\title{
Building an Evidence Base for Drug Abuse Treatment in Afghanistan: Lessons Learned and Implications for Future Research
}

Matthew Courser Ph.D. ${ }^{1}$ Knowlton Johnson Ph.D. ${ }^{1}$ Melissa H. Abadi Ph.D. ${ }^{l,}$ Stephen R. Shamblen Ph.D. ${ }^{1}$ Linda Young M.A. ${ }^{l}$ Kirsten Thompson, M.A. ${ }^{1}$ Thom Browne, M.A. ${ }^{2}$

${ }^{1}$ Pacific Institute for Research and Evaluation - Louisville Center, 1300 S. Fourth Street, Ste. 300 Louisville, KY 40208 U.S.A.

${ }^{2}$ United States Department of State: Bureau of International Narcotics and Law Enforcement, Washington, D.C. 20520 U.S.A.

\section{Abstract \\ Introduction}

Although, drug addiction is well documented in Afghanistan, organised drug abuse treatment (DAT) has been limited there until recently, and no evaluation studies of DAT outcomes in Afghanistan have been conducted. In response to this need, a feasibility study was conducted between October 2009 and January 2012 to evaluate the drug abuse treatment (DAT) model being implemented in Afghanistan, by the Colombo Plan for Cooperative Economic and Social Development in Asia and the Pacific (CPDAP). ${ }^{1}$

Two research questions are addressed by the evaluation study:

Q1. What is the pre-post change in treatment outcomes (drug use and criminal behaviour) for patients in the participating Afghan DAT Centers?

Q2. What factors explain changes in these outcomes?

\section{Methods}

A prospective cohort design was used to examine change in treatment outcomes in seven Afghan DAT centers that operate using the CPDAP treatment model. Four centers served males and three served females. A total of 504 in-person baseline interviews were conducted within 0 to 5 days of patients completing their detoxification process in the DAT centers. Baseline interviews were completed with 326 males and 178 females. On average, upon entry, male patients were 30.7 years old and female patients were 38.8 years old. Because the CPDAP treatment lasted up to 10 days of detox, 30-45 days of inpatient treatment, and 12 months of outpatient treatment, in-person posttest interviews were conducted 12 months after patients completed inpatient treatment. A total of 353 in person post-test interviews were conducted 13.5 months after DAT entry, yielding a 70\% study retention rate. 


\section{Results}

The study found that participation in DAT resulted in statistically significant decreases, in past 30-day use of any illegal drug, opiates, and alcohol. The results also showed statistically significant reductions, in the frequency of self-reported crime-related behaviour among patients from baseline to posttest. Finally, the study also found that completion of the first year of outpatient treatment, and completion of prior drug treatment predicted larger treatment effects.

\section{Discussion}

The findings of the present study suggest that DAT is effective in Afghanistan. As we have found that completion of the first year of outpatient treatment predicted positive treatment outcomes, DAT centers need to continue their emphasis on ensuring that patients complete both inpatient and outpatient treatment.

Our findings also suggest that drug testing - utilising newer technologies such as oral fluid assays - should be used at both pre-test and post-test to ensure accurate prevalence rates and to eliminate problems with false positives. Future studies will benefit from additional training of DAT center staff on how to collect treatment dosage information. Finally, future studies should consider the use of a comparison or control group to increase confidence that CPDAP treatment was the major cause of reductions in the prevalence of drug use and criminal behaviour.

Key words: Drug treatment in Afghanistan; Colombo Plan; Drug abuse in Afghanistan

\section{Introduction}

Over the past 30 years, a growing body of research has supported the effectiveness of Drug Abuse Treatment (DAT) in general, e.g., Simpson \& Curry $^{2}$;Gossop, ${ }^{3}$ and residential DAT programs in particular. ${ }^{2-9}$ Treatment outcome studies that have shown successful post-treatment outcomes for patients include the Treatment Outcome Prospective Study (TOPS), ${ }^{4,10}$ the Drug Abuse Treatment Outcome Study (DATOS) ${ }^{5}$ the National Treatment Improvement Evaluation Study (NTIES) ${ }^{11}$ and the National Treatment Outcome Research Study (NTORS). ${ }^{12}$ Additional research has suggested that contextual factors and organizational factors also influence treatment success. ${ }^{13}$ The study detailed below reports patient-level outcomes from an evaluation conducted between 2009 and 2012 of seven residential drug treatment centers in Afghanistan.

Drug use in Afghanistan is pervasive with one million drug addicts. ${ }^{14}$ According to the United Nations Office on Drugs and Crime (UNODC), approximately $8 \%$ of Afghans between 15 and 64 are drug abusers, which 
is about twice the global average. ${ }^{15}$ Moreover, there are indications that drug use in Afghanistan is underreported due to difficulties inherent in measuring a highly stigmatised and illegal behaviour. ${ }^{14}$ A more recent Afghanistan National Urban Drug Use Survey (ANDUS) estimated the number of drug users between 1.3 to 1.6 million. ${ }^{16}$ The same UNODC report indicated that although $11 \%$ of the sample had received DAT, more than $90 \%$ of adults surveyed indicated they needed treatment. ${ }^{15}$

Although drug addiction and use, especially opium, is well documented in Afghanistan, DAT in Afghanistan has been hardly documented till recently. ${ }^{14}$ In response to this need, the Bureau of International Narcotics and Law Enforcement Affairs (INL) of the United States Department of State is working with the Colombo Plan for Cooperative Economic and Social Development in Asia and the Pacific (CPDAP), to improve the availability and quality of DAT services in Afghanistan. By providing training to DAT centers, and developing a certification process for DAT Center staff, CPDAP has worked to bring evidence-based treatment practices to Afghanistan. ${ }^{16}$ In 2012, 398 Afghan DAT Center staff members were certified in the CPDAP treatment model. ${ }^{16}$

The CPDAP treatment model includes three main phases: 1) Pre-treatment, which is one to three months in length and focused on awareness (e.g., community and family environment/ support, community integration, and intake assessment) and motivation (e.g., motivational interviewing, crisis intervention, and counselling); 2) Treatment, which begins with 1 to 10 days in detoxification and is 1 to 35 days in length, with primary inpatient treatment (11-20 days), secondary inpatient treatment (21-30 days), and aftercare (3145 days), with the first two phases focussing on client education, counselling, vocational skills, religious sessions, recreation, and the third phase focusing on reintegration, counselling, recreation, self-help groups and employment; 3 ) Post-treatment, which includes two phases of outpatient treatment. Phase 1 of post-treatment consists of one year of counselling, self-help, and monitoring with varying levels of exposure, decreasing after the first three months, from once a week to twice a month, and then in the final six months from twice a month to once a month. Phase 2 of post-treatment consists of one year of counselling, visitation, drop-ins, and support groups once every three months. The present study evaluated the impact of the treatment phase and Phase I of the post-treatment phase.

Although, significant progress has been made in moving DAT in Afghanistan towards an evidence-based model, there have been no evaluation studies that report on outcomes of DAT in Afghanistan. This study reports the methodology and outcomes from an evaluation of seven DAT centers that operate using the CPDAP treatment model, as well as concomitant methodological challenges. 
Two research questions are addressed by the study:

Q1. What is the pre-post change in treatment outcomes (drug use and criminal behaviour) for patients in the participating Afghan DAT Centers?

Q2. What factors explain changes in these outcomes?

\section{Method}

\section{Research setting and study design}

The evaluation study was conducted between October 2009 and January 2012, in seven residential DAT centers trained in the CPDAP treatment model. These centers had the capacity to treat up to 1,452 patients, with 45days of residential treatment and two-years of outpatient treatment for drug addiction. Four of the centers evaluated, served males exclusively, and three served women and children exclusively.

A prospective cohort design was used to follow patients between treatment entry, detoxification, inpatient treatment, and completion of the first year of outpatient treatment. A baseline interview was completed within 0-10 days of the patient entering detoxification, and the post-test interview was completed 12 months after the patient completed inpatient treatment. Thus, the study employed a longitudinal design in which only an intervention group of treatment was followed over time. Practical limitations precluded the use of an experimental design or a quasi-experimental design using a comparison group.

The data for this study were collected by provincial field interviewing teams managed by the Afghan Center for Socio-Economic and Opinion Research (ACSOR) and ACSOR's parent company in the United States, D3 Systems, Inc. Because of Afghan cultural norms, all patient interviews and project data collection activities were completed by gender-matched field interviewing teams.

\section{Participants}

A total of 504 baseline in-person interviews were completed within 0 to 5 days of patients completing their detoxification process in the DAT centers which included 326 males (64\%) and 178 females (36\%). In-person posttest interviews were conducted and completed 12 months after the patient completed inpatient treatment, regardless of the length of stay in the 12-month outpatient treatment. A total of 353 post-test interviews were completed, representing a $70 \%$ study retention rate. Immediately following the post-test interview, patients completed urine tests to verify their self-reported use of benzodiazepines, marijuana, opiates, oxycodone and methamphetamine, the 
most commonly used illegal drugs in Afghanistan. These urine test verifications were completed with 341 of the 353 patients (97\%) who completed post-test interviews. It is important to note that $95 \%$ of patients completed the inpatient phase of treatment, while $82 \%$ completed the first year of the outpatient phase, representing high treatment completion rates.

\section{Human subjects protection}

Prior to the beginning the study, the interview questionnaires, research methods and procedures were reviewed and approved by the Institutional Review Board of the Pacific Institute for Research and Evaluation. A two-step informed consent process was employed, where patients first consented to the release of their names and admission dates to the research team, and then to participate in the study. Consent forms and interview questions were read aloud by the interviewers to the patients due to low literacy levels. Patients were informed that their participation was voluntary, that their decision on whether to participate would not result in any penalties or benefits to them or the Center, that their responses would be confidential, and that they could decline to answer any questions.

\section{Measures}

The evaluation focused on measuring changes in patient illegal drug consumption and alcohol use (past 30 days), criminal behaviour (serious crimes such as robbery, arson, and violence against others), less serious crimes such as forgery, buying and selling stolen property, and theft), and the number of times patients were arrested in the past 30 days. Pre-test measures of illegal drug consumption by patients came from patient self-reports on the pre-test interview, and post-test patient drug use was recorded, if the patient self-reported use in the post-test interview or indicated 'positive' in a urine test. Alcohol use (past 30 days), and criminal behaviour (past 30 days) were also measured, using self-report data from the pre and post-test interviews and also patient characteristics (e.g., gender, age, marital status and ethnicity).

The evaluation also collected data about the seven participating DAT Centers and its treatment (e.g. DAT Center staffing levels, number of days patients spent in each of the CPDAP treatment phases, patient treatment satisfaction, and patient participation in- and out-patient treatment activities).

\section{Analysis strategy}

Data analysis involved (a) data processing and data file creation (b) constructing scales, indexes, and new single item measures using principal component analyses, and reliability analyses (c) a Heckman ${ }^{17,18}$ two-step analysis to statistically control for attrition (or selectivity) biases (d) missing value imputation for potential covariates using EM algorithm imputation (e) 
identifying covariates that were correlated with outcomes and (f) conducting inferential analyses.

The inferential analyses examined changes over time on the outcomes of interest (Q-1), and predictors of changes in outcomes (Q-2). The primary analysis technique used for Q-1 was hierarchical (non-) linear modelling to model repeated measurements for patients, as this approach allows us to include cases without two repeated observations. Q-2 was addressed using logistic regression, where predictors that were significantly related to baseline standing were entered as predictors of positive changes due to the intervention [i.e., using at baseline ${ }^{1}$ and abstinent at follow-up vs. otherwise $(0)]$. Odds ratios were calculated as a measure of effect size, which are listed in the tables accompanying the analysis. Odds ratios less than one indicate desirable changes for Q-1 and odds ratios greater than one indicate a positive association between the named predictor and substance use reduction for Q-2. All inferential analyses were statistically controlled for selectivity due to attrition. ${ }^{17,18}$

\section{Results}

\section{Changes in illegal drug consumption}

Between the pre-test and post-test interviews, there were statistically significant decreases during the past 30 -day consumption of any illegal drug, opiates, and alcohol use by patients (Table 1 ).

\section{Changes in criminal behaviour}

As shown in Table 2, there were statistically significant reductions in the frequency of self-reported criminal behaviour among patients from baseline to post-test. These decreases in patterns of crime-related behaviour are small to medium size in effect. 
Table 1. Changes in illegal drug consumption and alcohol use, \% decrease and Odds Ratio (OR)

$\begin{array}{cccc}\text { Pre } & \text { Post } & \% & \text { Change OR } \\ \mathrm{N}=504 & \mathrm{~N}=353 & \text { Decrease } & \end{array}$

Drug Use Outcomes (drug test corrected)

any illegal drug - past 30 days

$\begin{array}{llll}85 \% & 75 \% & 12 \% & .46^{*}\end{array}$

benzodiazepines - past 30 days

$0 \quad 50$

$-$

.00

THC - past 30 days

6

45

opiates - past 30 days

84

58

31

$.23 *$

oxycodone - past 30 days

0

41

methamphetamine - past 30 days

0

Alcohol Use Outcomes

alcohol use - past 30 days

12

3

76

$.22 *$

binge alcohol use - past 30 days

4

0

100

.00

$* \mathrm{p}<.05,+\mathrm{p}<.10$; All comparisons were made using hierarchical non-linear modelling with a logit link function when controlling for selectivity biases due to attrition. For one outcome denoted by a range (e.g., 0-3), hierarchical linear modelling was used and the effect size ' $r$ ' is listed, as opposed to an odds ratio. A hyphen in a table cell indicates that a value was not calculated, either due to proportions not differing from zero or due to percentage change not being able to be calculated for continuous outcomes. 
Table 2. Changes in related problems among patients

\begin{tabular}{|c|c|c|c|c|}
\hline & $\begin{array}{l}\text { Pre \% } \\
(\mathrm{N}=504)\end{array}$ & $\begin{array}{l}\text { Post \% } \\
(\mathrm{N}=353)\end{array}$ & $\begin{array}{l}\% \\
\text { Decrease }\end{array}$ & $\begin{array}{l}\text { Change } \\
\text { OR }\end{array}$ \\
\hline $\begin{array}{l}\text { Serious crimes during the past } \\
\text { month }\end{array}$ & $13 \%$ & $8 \%$ & $40 \%$ & $.57^{*}$ \\
\hline $\begin{array}{l}\text { Violence against other persons } \\
\text { (homicide, aggravated assault, } \\
\text { kidnapping, etc.) }\end{array}$ & 2 & 1 & 67 & .35 \\
\hline $\begin{array}{l}\text { Sex offences (rape, aggravated } \\
\text { sexual assault) }\end{array}$ & 5 & 0 & 94 & $.05^{*}$ \\
\hline $\begin{array}{l}\text { Sale, distribution, growing, } \\
\text { farming or cultivation of any } \\
\text { drugs (not counting drug use or } \\
\text { possession) }\end{array}$ & 7 & 6 & 7 & .95 \\
\hline $\begin{array}{l}\text { Robbery (armed robbery, } \\
\text { mugging) }\end{array}$ & 2 & 1 & 67 & .32 \\
\hline Arson or weapons offenses & 1 & 1 & -33 & 1.31 \\
\hline $\begin{array}{l}\text { Crimes that are not serious during } \\
\text { the past month }\end{array}$ & 25 & 13 & 48 & $.45^{*}$ \\
\hline Forgery or fraud & 6 & 3 & 43 & .59 \\
\hline Buying/receiving stolen property & 4 & 5 & -19 & 1.23 \\
\hline Gambling & 13 & 11 & 20 & .77 \\
\hline Prostitution or pimping & 10 & 4 & 58 & $.38^{*}$ \\
\hline Burglary or auto theft & 4 & 1 & 72 & $.27^{*}$ \\
\hline Other theft (larceny, shoplifting) & 4 & 1 & 78 & $.22 *$ \\
\hline Vandalism, or loitering & 7 & 1 & 92 & $.08^{*}$ \\
\hline
\end{tabular}

* $\mathrm{p}<.05,+\mathrm{p}<.10$; All comparisons were made using hierarchical non-linear modelling with a logit link function when controlling for selectivity biases due to attrition. For the two continuous outcomes denoted by number of days, hierarchical linear modelling was used and the effect size ' $r$ ' is listed, as opposed to an Odds Ratio. A hyphen in a table cell indicates that a value was unable to be calculated, either due to proportions not differing from zero or due to percentage change not being able to be calculated for continuous outcomes. 
Table 3. Predictors of significant change in treatment outcomes $(n=353)$

\section{Patient Characteristics}

Males

Reduction during the past month - any illegal drug use (Odds Ratios)

Reduction during the past month Opiates Use

(Odds

Ratios)

Age in years

2.72

1.00

$3.84 \mathrm{E}+09$

Pashtun ethnicity

Turkmen ethnicity

.98

1.00

1.06

.75

1.03

.97

Years of education

.95

Worked towards high school diploma or voc.

degree

.88

.62

.03

.45

$.02+$

1.04

Learned to read

1.27

.92

Lived with family other than spouse prior to

treatment

.44

.94

.84

Prior drug treatment

Self-efficacy (0-3)

Stressful life events (0-8)

.80

$2.01 *$

1.04

.76

.94

Interview conducted in Dari language (Pashto otherwise)

\section{Patient treatment dosage and treatment}

\section{satisfaction}

Number of days in treatment (self-report)

Completed outpatient treatment

Inpatient treatment satisfaction (0-3)

Outpatient treatment satisfaction (0-3)

Days in inpatient treatment

Inpatient dosage--training (0-4)

1.01

$6.01 *$

$1.01+$

1.03

$4.84 *$

1.00

1.37

$1.33+$

.86

1.02

1.09

Inpatient dosage--job placement (0-1)

\section{Director/Center Characteristics}

Director involvement in treatment in hours

Salaried full time staff

Staff attending Colombo training

1.06

Staffing $(0-3)$

$* \mathrm{p}<.05,+\mathrm{p}<.10 ;$ All treatment implementation, context, individual characteristics and director/Centre characteristics were screened for a zero order relationship with either substance use variable. The variables listed here are the only variables having a zero-order relationship with substance use and positive substance use outcomes corrected by drug test results (i.e., use at pre-test and no use at post) were regressed on these variables using logistic regression. 


\section{Predictors of outcomes in treatment}

Research question 3 sought to understand the factors that predicted the positive changes in illegal drug use and criminal behaviour shown in Tables 1 and 2. Initially, a bivariate correlational analysis was conducted of 115 predictors with key patient outcomes. Table 3, which includes results from a logistic regression analysis, includes predictors that were statistically significant at the bivariate level.

\section{Discussion}

Our evaluation study demonstrated statistically significant positive changes in outcome, at the seven participating DAT centers. Using a prospective cohort design, the evaluation found preliminary evidence that completing the inpatient and outpatient components of the CPDAP treatment model, resulted in decreased prevalence of illegal drug and alcohol use as well as criminal behaviour.

Related to research question 1 , in the 30 days prior to treatment, $85 \%$ of patients reported using at least one illegal drug (benzodiazepines, drugs containing THC, opiates, oxycodone, or methamphetamine). At the time of the post-test interview, $75 \%$ of patients used at least one illegal drug, a 12\% decrease relative to the baseline prevalence. In the 30 days prior to treatment, $84 \%$ of patients reported using opiates. At the time of the post-test interview, $58 \%$ of patients reported using opiates, a $31 \%$ decrease relative to the baseline prevalence. In the 30 days prior to treatment, $12 \%$ of patients reported using alcohol. At the time of the post-test interview, 3\% of patients reported using alcohol, a 76\% decrease relative to the baseline prevalence. Two Illegal drugs had past 30-day prevalence rates that significantly increased between pre-test and post-test. Drugs containing tetrahydrocannabinol (THC) increased from $6 \%$ at baseline to $45 \%$ at post-test, and oxycodone use increased from $0 \%$ at baseline to $41 \%$ at post-test.

The reductions in opioid use are even more striking, given that previous research has found that opioid addicts have particularly low abstinence rates after inpatient treatment, with some studies reporting that only $15 \%$ of the patients, who could be found at six month follow up remained opioid free. ${ }^{19} \mathrm{~A}$ 1997 National Institutes of Health consensus statement on Effective Medical Treatment for Opiate Addiction stated that abstinence "cannot be achieved or sustained by the majority of opiate-dependent people." ${ }^{20}$ In their follow up study of 115 opioid addicts admitted to a VA inpatient detoxification program, which included therapy and an intensive outpatient programme with optional naltrexone medication, Davison and colleague ${ }^{21}$ reported that one year later, a fifth $(20.5 \%)$ of the sample had been readmitted to detoxification, while $23 \%$ had remained in outpatient treatment for at least 90 days and only three patients had provided consistently negative urine samples collected during that period. 
Our study also found statistically significant reductions in the frequency of self-reported crime-related behaviour among patients from baseline to posttest. The prevalence of self-reported serious crimes (such as robbery, arson, and violence against others) in the past month decreased from $13 \%$ at baseline to $8 \%$ at post-test, a $40 \%$ reduction relative to the baseline percentage. Prevalence of self-reported non-serious crimes (such as forgery, buying and selling stolen property and theft) (during the past month) has decreased from $25 \%$ at baseline to $13 \%$ at post-test, a $48 \%$ reduction relative to the baseline percentage. Self-reported arrests in the past six months decreased from $12 \%$ at baseline to $7 \%$ at post-test, a $46 \%$ reduction relative to the baseline percentage. These results are similar to what has been found for evaluations of other treatment models being implemented in Peru, El Salvador, and Thailand. ${ }^{7-9,24}$

Related to research question 2, we found that patients who completed outpatient treatment were six times more likely $(\mathrm{OR}=6.01)$ to have a reduction in any illegal drug use, and almost five times more likely $(\mathrm{OR}=4.84)$ to have a reduction in opiate use, from pre-test to post-test. In addition, patients who reported being admitted to drug treatment prior to entering a CPDAP Center were twice as likely $(\mathrm{OR}=2.01)$ to have a reduction in opiate use, from pre-test to post-test. These results are generally consistent with findings of previous evaluations. ${ }^{4,8,22,23}$ It should be noted that treatment dropout and attrition were not significant problems for the seven DAT centers included in this evaluation. The average patient length of stay (including outpatient treatment) was 361 days, suggesting that patients participated in almost all detox, inpatient, and outpatient treatment activities prescribed as part of the CPDAP treatment model.

\section{Study implications}

These results and our experience suggest a number of important policy and methodological implications that can inform future studies. We also discuss study designs for future research.

\section{Treatment implications}

The results of the present study suggest two implications for treatment practice in Afghanistan. First, our results suggest that the CPDAP treatment model has produced positive change among patients in the consumption of illegal drugs, alcohol use, and criminal activity in the seven DAT centers which participated in the study. Although, additional research that includes a larger number of DAT centers which utilises an experimental or quasi-experimental design is needed, our preliminary results support the continued utilisation CPDAP treatment model in Afghanistan. 
Secondly, because our study found that completion of the first year of CPDAP out-patient treatment predicted larger positive treatment outcomes, emphasis needs to remain on ensuring that patients complete out-patient treatment. Consideration should also be given to increasing the frequency of services offered in the second year of the CPDAP outpatient phase and providing technical assistance and monitoring to ensure that treatment dosage is uniform and is maximised across CPDAP DAT Centers.

\section{Methodological implications}

Our experience in the present study also suggests a number of methodological improvements that should be incorporated into future studies. First, our study experienced a much lower congruence rate between patient self-reports and drug tests (58\%) at post-test in Afghanistan, than in previous studies conducted in Peru, Thailand, and El Salvador (95\% or higher). ${ }^{8,9,24}$ The present study partially corrected this bias by defining use at the post-test, for any patient who indicated illegal drug use in the post interview or tested positive in the drug test. Future studies should employ drug testing at both pre-test and posttest to enhance reliability of illegal drug use measures, especially, research that indicates prevalence estimates of illegal drug use in Afghanistan which are particularly low, due to the stigmatised nature of their use. ${ }^{15}$

Secondly, the present study used urine field test kits as the primary methodology for drug testing. However, a number of substances can result in urine test kits reading a false positive, particularly for opiates, drugs containing THC and methamphetamine, which cannot be detected or corrected at the analysis stage. Future studies should utilise newer technologies such as oral fluid samples and lab-based drug testing technologies (e.g., oral fluid samples) and gas chromatography-mass spectrometry lab techniques (GC-MS), to avoid potential problems of false positives. ${ }^{25}$

Thirdly, a significant focus of the study involved collecting information about treatment dosage for individual patients using treatment log forms that DAT center staff completed the treatment log as patients progressed through the CPDAP detox, inpatient, and outpatient treatment phases. Center staff in our study struggled to complete the treatment logs accurately and the logs often had to be corrected or completely re-done after field staff review. Future studies should include in-person training on treatment log completion, along with ongoing monitoring and technical assistance by field teams to ensure accurate reporting. ${ }^{26}$

Finally, language orcultural issues impacted datacollectionand patientreporting in a number of instances. For example, when patient ages were compared from pre- to post-test, there were higher than expected inconsistencies between time points. Investigations by ACSOR (and confirmation by independent consultants) indicated that most Afghans do not celebrate or track birthdays. 
Thus, it is difficult to calculate their ages. We also found that patients were more likely to report being "jailed" than "arrested." We learned that it is not uncommon for Afghan families to confine or tie up addicts to prevent drug use, and Afghans perceive this, as "jail" even though there is no actual arrest. Future studies should conduct cognitive lab interviews with Afghans to help identify potential language or cultural issues in survey instruments, prior to data collection to identify and avoid such challenges. ${ }^{27}$

\section{Implications for future research}

Key limitations of the present study include a lack of a control or comparison group, that would allow us to better control confounding explanations for our findings, and treatment process data that was not as valid and reliable as we hoped. As a result, future research should incorporate the following study design features.

First, a comparison or control group design should be used to control potentially confounding explanations. Given the Afghan culture and context, and because there is currently no central intake point for patients entering treatment, it may not be feasible to implement a true experimental design at the patient level with random assignment to conditions. However, if the goal of a future study was to test the impact of enhancements to the CPDAP treatment model, DAT centers could be randomly assigned to experimental and comparison conditions with centers in one condition receiving a different treatment or a stronger dosage, (for example, longer planned length of stay in residential treatment), while the comparison condition continues to use the current planned length of stay in residential treatment. Other evidence-based alternative treatment processes might also be implemented, instead of a longer planned length of stay in centers assigned to the experimental condition.

If random assignment of DAT centers to conditions is not feasible, a quasiexperimental design, such as a nonequivalent control group design, might be implemented in which a center, implementing the CPDAP treatment model is compared to a group of centers using another treatment model. If there were a larger sample of centers, matching pairs of centers on relevant background characteristics, would help establish that CPDAP treatment was the major cause of any positive change over time.

Secondly, future studies should ensure that implementation fidelity protocols are designed and implemented to obtain detailed process data and to ensure close monitoring of the process data records. Detailed implementation data and monitoring will enhance the implementation of credibility of treatment components and activities, as well as facilitate fidelity analyses, which are critical given the cultural and ethnographic differences across Afghan provinces, cultural differences as to how male and female patients enter and proceed through treatment, and the fact that a variety of different non- 
government organisations operate the CPDAP DAT Centers. Moreover, collecting additional process data about implementation of CPDAP model components will make it easier to understand the relative impact of each phase of treatment and treatment activities on patient outcomes.

In conclusion, the results from this study are promising, and they support the need to conduct additional research to firmly establish the CPDAP treatment model as evidence-based. A common set of standards for evidence-based prevention and treatment interventions has been developed by Substance Abuse and Mental Health Administration's (SAMHSA) National Registry of Evidence-based Programs and Practices. We recommend that future evaluations of the CPDAP treatment model and drug abuse treatment in Afghanistan utilise designs and methodologies that conform to the requirements of the SAMHSA National Registry.

\section{Acknowledgements}

This research was supported by the U.S. Department of State, Bureau for International Narcotics and Law Enforcement Affairs, through contract number: S-AQMPD-067-D0116, K.W. Johnson, PI. We would like to thank the staff of D3 Systems \& Afghan Center for Socio-Economic and Opinion Research for their assistance in translation and data collection. We would also like to extend a special acknowledgement to the Afghan drug abuse treatment center directors and staff as well as the participants in this study. The views expressed in this paper do not necessarily reflect those of the U.S. Department of State.

\section{References}

1. The Colombo Plan: DAP Initiatives [Internet] 2011 [cited May 16, 2012] Available from: http://www.colombo-plan.org/index.php/programmes/ drug-advisory-programme/dap-initiatives

2. Simpson DD, Curry SJ. Special issue: drug abuse treatment outcome study (DATOS). Psychology of Addictive Behaviors 1997; 11: 211-337

3. Gossop M. The clinical fallacy and treatment outcomes. Addiction, 2008; 103: 89-90

4. Condelli WS, Hubbard RL. Relationship between time spent in treatment and resident outcomes from therapeutic communities - Journal of Substance Abuse Treatment 1994; 11: 25-33

5. Hubbard EL, Craddock SG, Flynn PM, Anderson J, Etheridge RM. Overview of 1-year follow-up outcomes in the Drug Abuse Treatment Outcome Study (DATOS) - Psychology of Addictive Behaviors, 1997; 11: 261-278 
6. Melnick G, Hawke J, Wexler, HK Client perceptions of prison-based therapeutic community drug treatment programs, The Prison Journal. 2004; 84(1): $121-138$

7. Johnson KW, Young L, Pan T, Zimmerman RS, Vanderhoff KJ. Therapeutic Communities (TC) drug treatment success in Thailand: A 2006 follow-up study,Research Monograph; 2007 Louisville, KY: Pacific Institute for Research and Evaluation —-Louisville Center

8. Johnson K, Pan Z, Young L, Vanderhoff J, Shamblen SR, Browne T, Suresh G. Therapeutic community drug treatment success in Peru: A follow-up outcome study, Substance Abuse Treatment, Prevention and Policy. 2008; 3(26): $1-15$

9. Johnson K, Young L, Shamblen SR, Suresh G, Browne T, Chookhare W. Evaluation of the Therapeutic Community treatment model in Thailand: Policy implications for compulsory and prison-based treatment, Substance Use and Misuse. 2012; 47: 889-909

10. Hubbard RL, Marsden ME, Rachal JV, Harwood HJ, Cavanaugh ER, Ginzburg HM, Drug Abuse Treatment: A National Study of Effectiveness. Chapel Hill: University of North Carolina Press, 1989

11. SAMHSA, NTIES. Substance Abuse and Mental Health Services Administration, Center for Substance Abuse Treatment. 1997, National Treatment Improvement Evaluation Study (NTIES), 1992-1997

12. Gossop M, Marsden J, Stewart D, Rolfe A. Treatment retention and 1 year outcomes for residential programmes in England, Drug and Alcohol Dependence, 1999; 57: 89-98.

13. Simpson DD, Flynn PM \& (Guest Editors), Organizational readiness for change. Journal of Substance Abuse Treatment. 2007; 33: 111-209

14. United Nations Office on Drugs and Crime. Drug use in Afghanistan: 2005 Survey - Executive Summary [Internet] 2011 [cited May 16, 2012]. Available from:http://www.unodc.org/documents/afghanistan//Counter_Narcotics/Afghanistan_Drug_Use_Survey_FINAL_Nov_2005.pdf

15. United Nations Office on Drugs and Crime [UNODC]. Drug use in Afghanistan: 2009 Survey - Executive Summary [Internet] 2010 [cited May 16, 2012] Available from : http://www.unodc.org/documents/data-and-analysis/Studies/Afghan-Drug-Survey-2009-Executive-Summary-web.pdf 
16. U.S. Department of State. (2012). Afghanistan National Urban Drug Use Survey (ANUDUS) - 2012. Washington, DC: U.S. Department of State, Bureau for International Narcotics and Law Enforcement Affairs. [Internet] 2010 [cited May 16, 2012] Available from: http:/www.colombo-plan.org/ index.php/acce-activities-2012

17. Heckman JJ. The common structure of statistical models of truncation, sample selection and limited dependent variables and a simple estimator for such models, Annals of Economic and Social Measurement. 1976; 5(4): 475-492

18. Heckman JJ. Sample selection bias as a specification error. Econometrica. 1976; 47(1): 153-161

19. Chutuape MA, Jasinski DR, Fingerhood MI, Stitzer, ML. One-, three-, and six-month outcomes after brief inpatient opioid detoxification. American Journal of Drug \& Alcohol Abuse. 2011; 27(1): 19

20. National Institute of Health. Effective medical treatment of opiate addiction. NIH Consensus Statement. 1997 November 17-19; 15(6): 1-38

21. Davison JW, Sweeney ML, Bush, KR, Correale TMD, Calsyn DA, Reoux JP, Kiviahan DR (2006). Outpatient treatment engagement and abstinence rates following inpatient opioid detoxification, Journal of Addictive Diseases. $2006 ; 25(4): 27-35$

22. DeLeon, G. The therapeutic community: Status and evolution, International Journal of Addictions. 1985. 20(6-7): 823-844

23. Coombs, R. H. Back on the street: Therapeutic communities' impact upon drug users. American Journal of Drug and Alcohol Abuse, 1981. 8: 185-201

24. Johnson, K, Shamblen, S, Courser, M, Young, L, Abadi, M, Browne, T. Drug use and treatment success among gang and non-gang members in El Salvador: a prospective cohort study, Substance Abuse Treat Prev Policy 2013;8(1):20

25. Moore L, Wicks J, Spiehler V, Holgate R. Gas chromatography-mass spectrometry confirmation of Cozart RapiScan saliva methadone and opiate tests, Journal of Analytical Toxicology. 2001; 25(7): 520-524

26. Rosa C, Campbell A, Kleppinger C, Sampson R, Tyso C, Mamay-Gentilin S. (2009). Quality assurance of research protocols conducted in the community: the National Institute on Drug Abuse Clinical Trials Network experience, Clinical Trials 2009;6(2): 151-161

27. Willis G. Cognitive interviewing, New York: Sage; 2004 